\title{
Applications of Inductive Learning to Automated Visual Inspection
}

\author{
Mehmet Sabih Aksoy \\ Department of Information Systems \\ College of Computer and Information Sciences \\ King Saud University \\ Riyadh, Saudi Arabia \\ Orhan Torkul \\ Departmen of Industrial Engineering \\ Sakarya University Faculty of Engineering \\ Sakarya, Turkey
}

\author{
Abdullah Almudimigh \\ Department of Information Systems \\ College of Computer and Information Sciences \\ King Saud University \\ Riyadh, Saudi Arabia \\ Ismail H. Cedimoglu \\ Department of Information Systems Engineering \\ Faculty of Computer and Information Science \\ Sakarya, Turkey
}

\begin{abstract}
In recent years, there has been a growing amount of research on inductive learning and its applications to different domains. Out of this research a number of promising algorithms have surfaced. Inductive learning algorithms are domain independent. In principle, they can be used in any task involving classification or pattern recognition. In this paper a number of applications of RULES family of induction algorithms to visual inspection are presented. The main advantages of using induction for visual inspection are: (a) The systems does not suffer from orientation problem which is very important for digital image processing. (b) The pattern does not have to be stored in the memory in graphics form because they are represented by rules. This saves memory space. (c) The decision can be reached in short time because the number of conditions in each rule and the total number of rules are not big. (d) It is easy to develop a software and design a hardware for these systems as they are not complicated.
\end{abstract}

Keywords: Induction, Inductive Learning, RULES3, RULES3-EXT, Expert Systems, Visual Inspection.

\section{INTRODUCTION}

In recent years, there has been a growing amount of research on inductive learning [1]. In its broadest sense, induction (or inductive inference) is a method of moving from the particular to the general - from specific examples to general rules [2]. Induction can be considered the process of generalising a procedural description from presented or observed examples [3].

The purpose of inductive learning is to perform a synthesis of new knowledge, and this is independent of the form given to the input information [4]. In order to form a knowledge base using inductive learning, the first task is to collect or establish a set of examples of expert decisions. Each example belongs to a known class that is described in terms of a number of attributes. These examples may be specified by an expert as a good tutorial set, or may come from some neutral source such as an archive, or can be formed as shown in different applications described in this study. The induction process will attempt to find a method of classifying an example, again expressed as a function of the attributes, that explains the training examples and that can also be used to classify previously unseen cases [5].

Inductive learning algorithms are domain independent. In principle, they can be used in any task involving classification or pattern recognition [6]. There have been several successful applications of RULES family of inductive learning systems. Some applications of inductive learning algorithms for automated visual inspection are summerized in this paper.

The organization of the paper is as follows: In section 2, RULES family of inductive learning algorithms and induction process of RULES3-EXT is outlined. Section 3 briefly explains visual inspection techniques. The applications of RULES family of algorithms to automated visual inspection are summarized in section 3 , and section 4 is the conclusion.

\section{RULES FAMILY OF INDUCTIVE LEARNING ALGORITHMS}

The first member of RULES family of algorithms RULES-1 [7], has been published in 1995. After that RULES2, RULES3, RULES3-Plus, RULES4, RULES5 [8], RULES6 [9], REX [10] and RULES3-EXT [11] have been developed.

RULES family of algorithms are used for extracting a set of classification rules from a collection of examples for objects belonging to one of a number of known classes. An object must be described in terms of a fixed set of attributes, each with its own range of possible values, which could be nominal or numerical. For example, attribute "length" might have nominal values \{short, medium, long\} or numerical values in the range $\{-10,10\}$.

An attribute-value pair establishes a condition in a rule. If the number of attributes is $\mathrm{Na}$, a rule might contain between one and $\mathrm{Na}$ conditions. Only conjunction of conditions is allowed in a rule and therefore the attributes must be all different if the rule contains more than one condition.

The main steps which describe the induction process of RULES3-EXT algorithm (the latest version of the Family) are given below. RULES3-EXT extracts rules by considering one example at a time. It forms an array consisting of all attribute-value pairs associated with the object in that example, the total number of elements in the array being equal to the number of attributes of the object. The rule 
forming procedure may require at most $\mathrm{Na}$ iterations per example. The algorithm can be summarized as follows:

Step 1. Define ranges for the attributes, which have numerical values and assign labels to those ranges

Step 2. Set the minimum number of conditions (Ncmin) for each rule

Step 3. Take an unclassified example

Step 4. Nc=Ncmin-1

Step 5. If $\mathrm{Nc}<\mathrm{Na}$ then $\mathrm{Nc}=\mathrm{Nc}+1$

Step 6. Take all values or labels contained in the example

Step 7. Form objects, which are combinations of Nc values or labels

taken from the values or labels obtained in Step 6

Step 8. If at least one of the objects belongs to a unique class then form

rules with those objects; ELSE go to Step 5

Step 9. Select the rule, which classifies the highest number of examples

Step 10. Remove examples classified by the selected rule Step 11. If there are no more unclassified examples then STOP;

\section{ELSE go to Step 3}

Here $\mathrm{Nc}$ is the number of condition(s) for each rule and $\mathrm{Na}$ is the number of attributes for each example.

\section{VISUAL INSPECTION TECHNIQUES}

There are many techniques for visual inspection (Davies, 1997; Jain et al., 1995). Four of them are (1) Image subtraction, (2) Dimensional verification, (3) Syntactic approach and, (4) Feature Matching (Roland and Charles, 1982).

In Image subtraction, the image to be inspected is scanned and compared against the original image, which has been stored before. The subtracted image is analyzed. This method needs large reference data storage, accurate alignment and sensitive illumination and scanner conditions. Also many images may not match point-by-point identically even when they are acceptable.

In Dimensional Verification Method the task is to make a determination for each measurement as to weather it falls within the previously established standards. The distance between edges of geometric shapes is the primary feature of this inspection method.

Syntactic Approach for inspection uses descriptions of a large set of complex objects using small sets of simple pattern primitive and structural rules. Primitives are small number of unique elements, such as lines or corners. A structural description of the primitives and the relationships between them can be determined to form a string grammar.

In Feature Matching or Template Matching method, the image to be inspected is scanned and the required features are extracted. Then these features are compared with those defined for the perfect pattern. This method greatly compresses the image data for storage and reduces the sensitivity of the input intensity data. A number of predefined binary templates can be used to extract the necessary features for images to be inspected.

For all applications, Feature (template) Matching Method is used because of its advantages mentioned above. Also this method is very suitable for RULES family of algorithms, as they need a set of examples which contain a number of attribute-value pairs and a class. Each template (mask) can be considered as an attribute and the frequencies of templates can be considered as values for those attributes. The class is the labeled image being processed.

\section{APPLICATIONS OF RULES FAMILY OF ALGORITHMS ON VISUAL INSPECTION}

Inductive learning algorithms are domain independent. In principle, they can be used in many tasks involving classification or pattern recognition [6]. There have been several successful applications of RULES family of algorithms on visual inspection. Some of them are summerized below.

\subsection{An Industrial Visual Inspection System}

A system that uses RULES3 induction algorithm has been developed for industrial visual inspection. The system uses RULES3 to extract the necessary set of rules and template matching technique for feature extraction. 20, 3x3 masks are used to represent an image (see Figure 1). Each example consists of 20 frequencies of each mask. The system was tested on five different types of tea or water cups in order to classify the good and bad items. It was trained using five good cups (see Figure 2) and then tested for 113 unseen examples. The results obtained showed the high performance of the system: the efficiency of the system for correctly classifying unseen examples was $\% 100$. The system can also decide what the type of the cup being processed is [12].

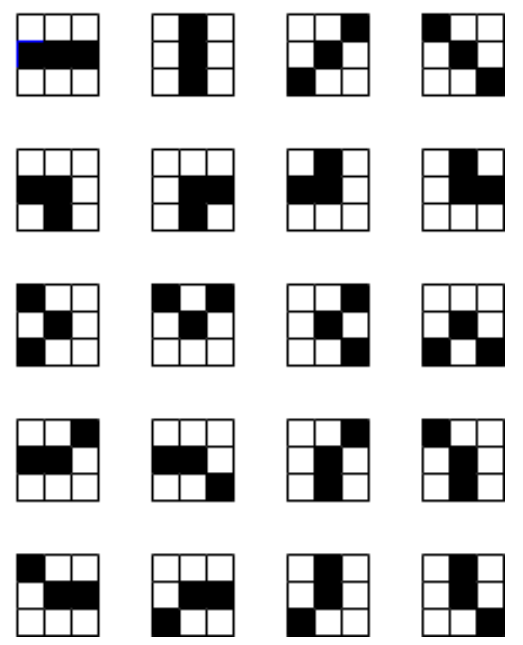

Fig 1: All possible 3x3 Masks

The system needs to learn the good cups. Each cup has to be presented as an example for the system to extract the necessary rules. In order to form an example the edges contained in each pattern are detected. In this study the Laplacian edge detection operator was chosen to be used because of its good performance among the 
edge detection operators (such as Sobel, Gradient and Robert) tested for the given five cups.

The system can also decide what the type of the cup is. This is done by calculating the difference of the frequencies of each mask for the pattern being processed and the frequencies of the given five patterns. The following formula (that is the sum of the squares of deviation) can be used for this purpose:

$D_{i}=\sum_{j=1}^{20}\left(F_{j}-F_{i j}\right)^{2}$

where $i=1,2,3,4,5$

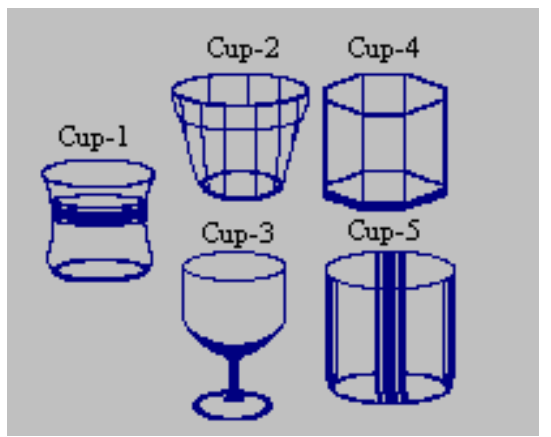

Fig 2: Five example good cups

Here $F_{j}=$ The frequency of the mask $M_{j}$ for the pattern being processed. pattern

$F_{i j}=$ The frequency of the mask $M_{j}$ for the $i^{\text {th }}$ good

The minimum D (that is the least square deviation) is chosen to decide what the type of the cup should be. For example if the minimum $\mathrm{D}$ is $\mathrm{D}_{2}$ that means the type is Cup-2.

\subsection{Banknote Recognition}

A system has been developed for this purpose. The system employs template matching for feature extraction and RULES3 to extract the necessary set of rules and to recognize a banknote. 10 of $3 \times 3$ masks were used to represent a banknote. Each banknote (or example) is presented by the frequencies of the masks used. The system was trained for all back and front sides of different Turkish and Saudi banknotes. Ten images were used for each side of the banknotes for learning purpose. The system has been tested using many "unseen" examples and correctly classified all of them $[13 ; 14]$.

\subsection{Signature Verification}

An alternative technique for Signature verification has been developed. The technique employs template matching for feature extraction and RULES3-EXT inductive learning algorithm to extract the necessary set of rules and to verify a signature. 15 of $3 \times 3$ masks were selected to represent a signature. Each signature (or pattern) is presented by the frequencies of the masks used. The system was trained using 144 signatures (16 signatures belonging to 9 different persons). The system has been tested using many unseen signatures and the ability to correctly classify them was found to be $97 \%$ [15].

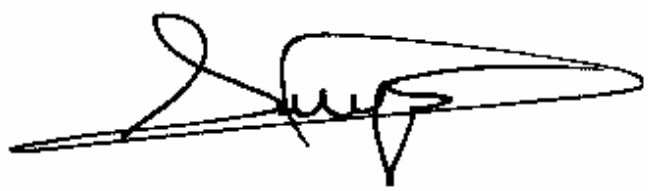

(a) A Siganture before edge detection

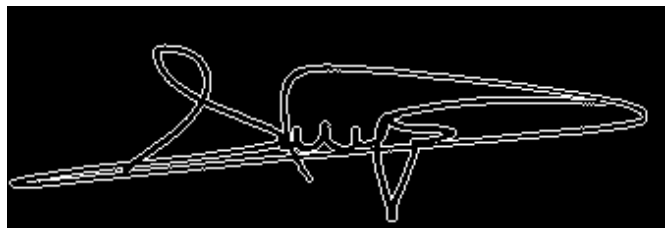

(b) A Signature after edge detection

Fig 3: A Signature before and after edge detection operation

\subsection{Numberplate Recognition}

An alternative technique has been developed for numberplate recognition. The technique uses RULES3 algorithm for learning and template matching technique for feature extraction. Each character (letter and number) that can be used in number-plates is considered as an example which consists of 20 frequencies of each $3 \times 3$ mask used for representation. In order to recognize a number-plate (see Figure 4), all characters contained are recognized one by one. After bringing them together the number-plate being processed is recognized [16].

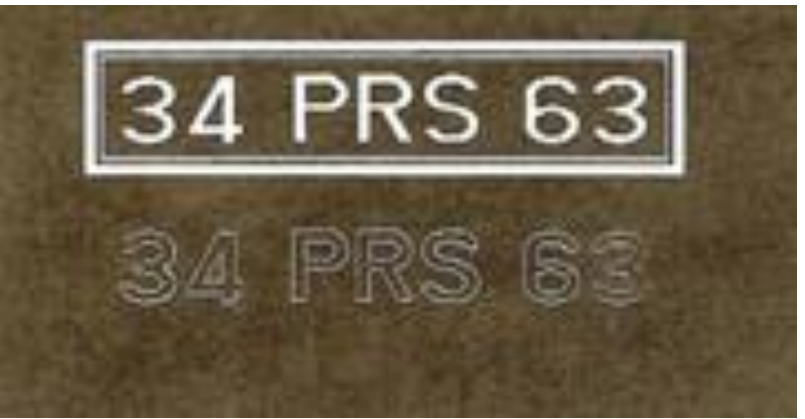

Fig 4: A Numberplate before and after edge detection operation

\subsection{Inspection of Ceramic Tiles}

In quality control process of ceramic tiles the aim is to figure out if the product is good or defective. In order to get detailed control information defected products are defined as "spots" and "cracks". If there are some spots and/or cracks on the product the system learns it as spot or crack, otherwise the product is accepted to be good. Some examples of good, spot and crack ceramic tiles are shown in (Figure 5. ) 


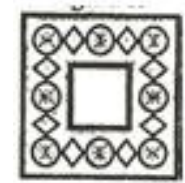

Good

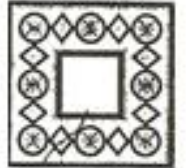

Crack

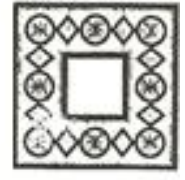

Spot
Fig 5: Three examples of tiles

The system first converts the image of tile to be processed to gray-level image if it is not. Then it increases the contrast of gray-level. For noise reduction the system uses median filtering method. The image then is coverted to a binary image using a threshold value. The edges contained in the binary image is obtained using Sobel edge detection operator. Finally the selected 10, 3x3 mask (see Figure 6) are applied to image being processed and their frequencies are calculated. After all examples have been processed RULES3 is used to extract e set of rules to be used for classification.

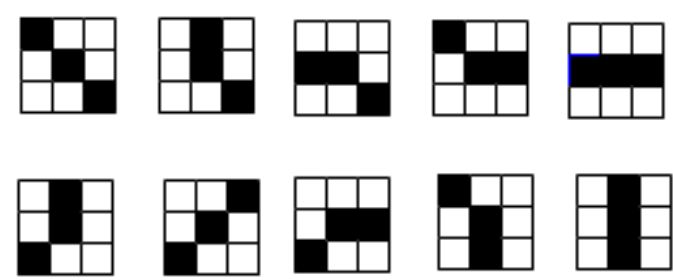

Fig 5: Selected 10 masks used for the study

More than 100 defected tiles (having crack and/or spot) and not defective tiles have been used for training. The efficiency of the system for correctly classifying many unseen examples was found to be $96 \%$. The details could be found in [17].

\subsection{Barcode Recognition}

A new technique has been developed for Barcode Recognition. The technique uses RULES3 system. In the technique only the tickness of vertical lines are considered while the spaces in between are ignored. Each line is considered an attribute and its tickness is considered as its value. For each barcode a rule is extracted using RULES3. No special hardware is required for the technique, only a PC and a barcode reader is enough. [18].

\section{CONCLUSION}

The induction of rules from empirical data is a useful technique for automatic knowledge acquisition. It offers a modularised, clearly explained format for decision making which is compatible with human reasoning procedures. Also, the resulting rules are suitable for use in expert systems. In recent years, many task-oriented inductive learning systems have been developed that have demonstrated impressive performance in their specific domain of application. This paper presents some applications of RULES3 and RULES3EXT inductive learning systems in Automated Visual Inspection. The main advantages of using induction for visual inspection are given below: (a) The orientation is a very important problem for digital image processing. In the applications shown in this study because of using template matching technique this problem has no effect. (b) Each pattern is represented by a rule. So the pattern does not have to be stored in the memory in graphics form. This saves memory space. (c) As the number of conditions in each rule and the total number of rules are not big, the decision can be reached in short time. (d) It is easy to develop a software and design a hardware for these system as yhet are not complicated.

However, some problems still remain. Most systems lack generality and extensibility. The future work should consentrate on these two issues.

\section{ACKNOWLEDGEMENT}

The authors would like to thank Research Center in King Saud University, College of Computer and Information Sciences, for its support to complete this study.

\section{REFERENCES}

[1] Nakakuki Y., Koseki Y. and Tanaka M. 1990 Inductive learning in probabilistic domain. In proceedings Eighth National Conf. on AI. Boston. pp.809-814.

[2] Forsyth R. 1989 Machine Learning principles and techniques. Ed: R. Forsyth, Chapman and Hall, London.

[3] Rubin S.H. 1991 Expert systems for knowledge acquisition. In proc. First World Congress on Expert Systems , Vol 3, Orlando, Florida, December 16-19, pp.1793-1799.

[4] Kodratoff Y. 1988 Introduction to machine learning", Pitman Publishing, London.

[5] Quinlan J.R. 1988 Induction, knowledge and expert systems. In Artificial Intelligence Developments and Applications. Eds J.S. Gero and R. Stanton, Amsterdam, North-Holland, pp.253-271.

[6] Liu W.Z. and White A.P. 1991 A review of inductive learning. In proc. Research and Development in Expert Systems VIII, Cambridge, pp.112-126.

[7] Pham D.T. and Aksoy M.S. 1995 RULES: A simple rule extraction system. Expert Systems with Applications, Vol.8, No.1, pp.59-65, USA.

[8] Aksoy, Mehmet Sabih. 2008 Review of RULES Family of Algorithms. Mathematical \& Computational Applications An International Journal, Vol.13 , No.1., pp.51-60.

[9] Pham D.T. and Afify A.A. 2005 RULES-6: A simple rule induction algorithm for handling large data sets. In Proc. Inst. Mech. Engs, Part C: J. Mech. Eng. Sci., vol.219, no.10, pp. 1119-1137.

[10] Akgöbek Ömer, Aydin Yavuz Selim, Öztemel Ercan and Aksoy Mehmet Sabih. 2006 A new algorithm for automatic knowledge acquisition in inductive learning. Knowledge-Based Systems, Volume 19, Issue 6, Pages 388-395.

[11] Mathkour H.I. 2010. RULES3-EXT Improvements On Rules-3 Induction Algorithm", Mathematical and Computational Applications, V.15, No.3.

[12] Aksoy M.S., Torkul O. and Cedimoglu I.H. 2004 An Industrial Visual Inspection System That Uses Inductive 
Learning. Journal of Intelligent Manufacturing, 15, pp:569-574.

[13] Sevkli M., Turkyilmaz A. and Aksoy M.S. 2002 Banknote recognition using inductive learning. Int. Conf. On Fuzzy Syst. And Soft Computational Intelligence in Management and Industrial Eng., FSSCIMIE'02, İstanbul Technical Univ., pp.122-128, Turkey, May 2931.

[14] Aksoy M.S. 2004 Saudi Banknote Recognition Using Inductive Learning. In proc. of $2^{\text {nd }}$ IIEC, December 1921, Riyadh, S.A.

[15] Aksoy, Mehmet Sabih \& Mathkour, Hassan. 2011 Signature Verification Using Rules3-Ext Inductive Learning System. International Journal of the Physical Sciences Vol. 6(18), pp. 4428 - 4434.
[16] Aksoy M.S., Cagil G. and Turker A.K. 2000 Numberplate recognition using inductive learning. Robotics and Autonomous Systems, (33), pp.149-153, Canada.

[17] Turkyilmaz A., Sevkli M. and Aksoy M.S. 2002 Inspection of Ceramic Tiles Using Inductive Learning. In pProc. $2^{\text {nd }}$ Intern. Conf. Responsive Manuf., ICRM, Univ. of Gaziantep, Turkey.

[18] Aksoy, M.S. and Bayram M.1996 A new technique to process and recognize barcodes using inductive learning. International Journal of Mathematical and Computational Applications, Vol.1, No.2, pp.1-6. 